Original Research Article

\title{
Linking of different ethnicities, races and religions to lipid profile patterns and hypolipidaemic drug usage patterns in coronary artery disease patients
}

\author{
Sanket P. Bharadwaj ${ }^{1}$, Aman T. Patel ${ }^{2}$, Devang A. Rana ${ }^{3 *}$, \\ Supriya D. Malhotra ${ }^{3}$, Tejas M. Patel ${ }^{4}$
}

${ }^{1}$ Student ${ }^{2}$ Department of Medicine ${ }^{3}$ Department of Pharmacology ${ }^{4}$ Department of Cardiology, Smt. NHL Municipal Medical College, Ahmedabad, Gujarat, India

Received: 13 June 2019

Revised: 30 June 2019

Accepted: 06 July 2019

*Correspondence to:

Dr. Devang A. Rana,

Email: devangandu@gmail.com

Copyright: (C) the author(s), publisher and licensee Medip Academy. This is an openaccess article distributed under the terms of the Creative Commons Attribution NonCommercial License, which permits unrestricted noncommercial use, distribution, and reproduction in any medium, provided the original work is properly cited.

\begin{abstract}
Background: Coronary artery disease (CAD) is the consequence of atherosclerosis in which inadequate blood flow in the coronary arteries leads to myocardial necrosis. The impact of ethnic on CAD might be underestimated within Indian communities. There have never been any studies done associating them to lipid profile patterns in the Indian setup hence this study is the first of its kind to work towards attending the absence of data in this direction.

The study aimed to evaluate the presence of ethnic differences in lipid profile patterns and hypolipidemic drug use in CAD patients.

Methods: An 8-week cross-sectional prospective study was conducted in the cardiology OPD of a tertiary care hospital. Adult CAD patients prescribed with at least one hypolipidaemic drug, having their lipid profile values and willing to give informed consent were selected. The prescription pattern was noted, and the lipid profile values of the patients classified as per ATP III guidelines by NCEP. Atherogenic dyslipidaemia was considered when patients had triglyceride levels $>150 \mathrm{mg} / \mathrm{dl}$ and HDL<40 mg/dl. The collected data was analyzed using SPSS. P value less than 0.05 was considered as statistically significant.

Results: A total of 123 patients enrolled. Out of these, 115 were Hindus and among Hindus, most were Brahmins (34). The most prescribed hypolipidaemic drug was Rosuvastatin. Thirty six patients had high triglyceride levels out of which 35 were Hindus. Low HDL (<40 mg/dl) was present in 70 patients out of which 64 were Hindus. Atherogenic dyslipidaemia was seen in 44 patients. Majority of them belonged to the age group of 51-60 years (43.2\%) and were Patels. Total cholesterol and LDL were high in 1 and 2 Jains respectively. Lipid values were higher in Tier-3 city patients.

Conclusion: Hindu patients in this study showed a poorer lipid profile while among the castes, Jains and Patel's fared poorly. It was seen that atherogenic dyslipidemia is on a rise in the Indian population.
\end{abstract}

Keywords: Caste, Coronary artery disease, Ethnicity, Hypolipidaemic drugs, Lipid profile patterns, Religion

\section{INTRODUCTION}

Coronary artery disease (CAD) is a group of diseases including stable and unstable angina, myocardial infarction and sudden cardiac death. ${ }^{1}$ It is the consequence of atherosclerosis in which inadequate blood flow in coronary arteries leads to myocardial necrosis. ${ }^{2}$
Modifiable risk factors like hypercholesterolaemia, hypertension, smoking, obesity, diabetes mellitus and sedentary lifestyle provide opportunities for individuals and populations to reduce their likelihood of developing CAD. Lipid profile, a screening tool for lipid abnormalities, typically includes Low-density lipoprotein (LDL), High-density lipoprotein (HDL), triglycerides 
(TG), total cholesterol and Very Low-density lipoprotein (VLDL). Most laboratories directly measure total cholesterol, HDL and triglycerides but LDL is calculated indirectly by the Friedewald equation: $\mathrm{LDL}=\mathrm{TC}-\mathrm{HDL}-$ (TG/2.2) (all in mmol/l) where TG/2.2 approximates to VLDL levels. ${ }^{3}$ This method is not appropriate for individuals with TG levels $>4.5$.

Atherosclerosis is mainly associated with increased LDL and decreased HDL levels in blood. ${ }^{4}$ Hypolipidemic drugs like statins, Ezetimibe, bile acid sequestrants, Fibrates and Niacin are used for dyslipidaemia. Nutritional status, food practices, preparation techniques, meal formats, resource availability, healthcare access, educational and economic status have ethnic differences. Race presumes shared genetic and geographical traits whereas ethnicity indicates shared cultural, historical, linguistic and religious traits. So, individuals of the same racial background are likely to carry more common genetic architecture than those belonging to the same ethnicity. The burden of CAD in India is huge and is a cause of concern due to manifestations at an earlier age as well as more severity and poorer prognosis than Western populations. ${ }^{5}$

\section{METHODS}

This cross-sectional prospective study began after obtaining permission from the Institutional Ethics Committee. The patients were informed that their participation is entirely voluntary, and their written consent was obtained in vernacular language. Confidentiality of data was maintained. The study was conducted in the cardiology OPD of a tertiary care hospital for a period of 8 weeks.

\section{Criteria for selection}

Adult CAD patients prescribed with at least one hypolipidaemic drug, having their lipid profile values and willing to give informed consent for the study were selected.

\section{Case record form}

A case record form was used to note down the ethnic details among which religion, caste and geographical distribution were dealt with in detail. The prescription pattern of the patients was recorded. The lipid profile variables of the patients were also collected from laboratory reports and recorded. Risk assessment by ATP (Adult Treatment Panel) III guidelines by NCEP (National Cholesterol Education Program). ${ }^{6}$ The lipid profile values of the patients in this study were classified and compared as per these guidelines. Atherogenic dyslipidaemia was considered as present when patients had triglycerides $>150$ $\mathrm{mg} / \mathrm{dl}$ along with HDL<40 mg/dl.

\section{Statistical analysis}

The collected data was entered in Microsoft Excel, 2016 version and was analyzed using SPSS (Statistical Package for
Social Sciences) version 23.0 (IBM Corporation, California). Pearson Correlation Coefficient test was used. Unpaired t-test was used to compare the ethnic parameters. $P$ value less than 0.05 was considered as statistically significant.

\section{RESULTS}

\section{Sociodemographic details}

A total of 123 patients were enrolled in the study. The mean \pm SD for age was $60.5 \pm 9.85$ years. The male: female ratio was $4.9: 1.115(93.5 \%)$ patients were Hindus and they were further subdivided according to their social caste. Majority of them were Brahmins (27.64\%). The patients belonged to 28 cities across 4 states of India: Gujarat, Rajasthan, Maharashtra and Madhya Pradesh. Majority of the patients belonged to Gujarat. For the sake of simplicity in comparing the data, the cities to which the patients belonged were grouped as Tier-1, 2 and 3, with 53, 28 and 42 patients respectively.

\section{Evaluation of drug utilization patterns}

A total of 485 drug formulations were prescribed to 123 patients. The mean number of drugs per prescription \pm SD was 3.94 \pm 1.12 . While for fixed dose combinations the value was $1.2 \pm 0.84$. Table 1 shows hypolipidaemic drugs and their combinations prescribed. In case of nonhypolipidaemic drugs, the most commonly prescribed drug formulation was Aspirin with Clopidogrel, in 80 patients.

\section{Evaluation of lipid profile patterns}

The mean \pm SD for total cholesterol was 136.14 \pm 37.04 $\mathrm{mg} / \mathrm{dl}$ (desirable). Only one patient had high total cholesterol $(>240 \mathrm{mg} / \mathrm{dl})$. The mean \pm SD for triglycerides was $170.32 \pm 76.81 \mathrm{mg} / \mathrm{dl}$ (borderline high). $53.7 \%$ patients had triglyceride levels $>150 \mathrm{mg} / \mathrm{dl}$. The mean \pm SD for HDL was $39.76 \pm 9.46 \mathrm{mg} / \mathrm{dl}$ (low). Low HDL (<40 mg/dl) was present in $70(56.91 \%)$ patients. The mean \pm SD for LDL was $62.92 \pm 30.28 \mathrm{mg} / \mathrm{dl}$ (optimal). The mean $\pm \mathrm{SD}$ for VLDL was $34.01 \pm 15.39 \mathrm{mg} / \mathrm{dl}$ (normal). 36(29.3\%) patients had higher VLDL levels.

\section{Atherogenic dyslipidaemia}

It was seen in $35.77 \%$ patients. Majority of them belonged to the age group of 51-60 years (43.2\%).

\section{Ethnicity and its association with lipid values and hypolipidemic drug utilization patterns}

\section{Religion}

As shown in Table 2, high triglyceride levels were seen in 35 Hindus and 1 Muslim patient. Total cholesterol was found to be high/borderline high in 6 Hindu patients. Very high LDL levels were found in 2 Hindu patients. High VLDL levels were found in 35 Hindus and one Muslim 
patient. 39 Hindus, 3 Muslims, 1 Sikh and 1 Christian patient had atherogenic dyslipidaemia.

\section{Caste}

The mean \pm SD for total cholesterol was the highest and the lowest in Jains and Vaishnavas respectively. (Table 3) Only 1 Jain patient had total cholesterol $>240 \mathrm{mg} / \mathrm{dl}$. Only 2 Jains had LDL $>190 \mathrm{mg} / \mathrm{dl}$. Atherogenic dyslipidemia was found to have maximum prevalence in Patels.

\section{Geographical distribution}

As shown in Table 4, the mean $\pm \mathrm{SD}$ is the lowest for patients in Tier-3 cities i.e. the disease engulfs them at a younger age. Most lipid values are higher in patients of Tier-3 cities. Majority of the elderly patients belonged to Tier-1 cities. Atorvastatin was prescribed more to the patients from Tier-3 cities. The number of patients with atherogenic dyslipidaemia residing in Tier-1, Tier-2 and Tier-3 cities was 20, 9 and 15 respectively.
- Tier-1: Ahmedabad

- Tier-2: Bhavnagar, Gwalior, Jaipur, Jamnagar, Jodhpur, Rajkot, Surat, Vadodara

- Tier-3: Others

\section{Determination of age as a major risk factor}

89 males $(72.36 \%)$ and 17 females $(13.82 \%)$ were more than 45 years and 55 years of age respectively.

\section{Correlation}

Using the Pearson correlation test, correlation between age and number of drugs was significant at the 0.01 level (2tailed) showing that more number of drugs are prescribed with increasing age. Unpaired t-test was used to compare various parameters on ethnic basis. Results shown in Table 5 were significant/extremely significant. ( $P$ value less than 0.05 and 0.001 respectively).

Table 1: Hypolipidemic drugs and drug combinations ( $N=123)$.

\begin{tabular}{|lll|}
\hline Hypolipidaemic drug/FDC & Number of patients prescribed & Percentage \\
\hline Rosuvastatin & 82 & 66.67 \\
\hline Atorvastatin & 29 & 23.58 \\
\hline Atorvastatin+Aspirin & 5 & 4.07 \\
\hline Rosuvastatin+Fenofibrate & 3 & 2.44 \\
\hline Atorvastatin+Clopidogrel+Aspirin & 1 & 0.81 \\
\hline Atorvastatin+Clopidogrel & 1 & 0.81 \\
\hline Rosuvastatin+Clopidogrel+Aspirin & 1 & 0.81 \\
\hline Total & 123 & 100 \\
\hline
\end{tabular}

Table 2: Mean \pm SD and $P$ values for various variables in patients of different religions.

\begin{tabular}{|llllll|}
\hline Religion & $\begin{array}{l}\text { Triglycerides } \\
(\mathbf{m g} / \mathbf{d l})\end{array}$ & $\begin{array}{l}\text { Total cholesterol } \\
(\mathbf{m g} / \mathbf{d l})\end{array}$ & HDL $(\mathbf{m g} / \mathbf{d l})$ & LDL (mg/dl) & VLDL (mg/dl) \\
\hline Hindus & $171.79 \pm 78.58$ & $136.83 \pm 37.25$ & $39.88 \pm 9.58$ & $63.23 \pm 30.28$ & $34.30 \pm 15.74$ \\
\hline Non-hindus & $149.17 \pm 41.11$ & $126.21 \pm 34.46$ & $37.85 \pm 7.7$ & $58.53 \pm 32$ & $29.83 \pm 8.2$ \\
\hline P value & 0.4228 & 0.4352 & 0.5593 & 0.673 & 0.429 \\
\hline
\end{tabular}

*Unpaired t-test was used. $\mathrm{P}$ value $<0.05$ was considered as statistically significant.

Table 3: Caste distribution of total cholesterol levels.

\begin{tabular}{|llllll|}
\hline Caste & $\mathbf{N}$ & $\mathbf{M e a n} \pm \mathbf{S D}(\mathbf{m g} / \mathbf{d l})$ & $<\mathbf{2 0 0}$ & $\mathbf{2 0 0 - 2 3 9}$ & $\mathbf{> = 2 4 0}$ \\
\hline Brahmin & 34 & $139.42 \pm 29.52$ & 32 & 2 & 0 \\
\hline Patel & 27 & $137.76 \pm 40.13$ & 25 & 2 & 0 \\
\hline Rajput & 21 & $127.73 \pm 31.88$ & 21 & 1 & 0 \\
\hline Jain & 12 & $148.31 \pm 68.58$ & 10 & 0 & 0 \\
\hline Vaishnava & 7 & $123.12 \pm 26.03$ & 7 & 0 & 0 \\
\hline Prajapati & 4 & $134.62 \pm 26.86$ & 4 & 0 & 0 \\
\hline Rabari & 3 & $157.86 \pm 28.23$ & 3 & 0 & 0 \\
\hline Jat & 2 & $126.95 \pm 5.30$ & 2 & 0 & 0 \\
\hline Sindhi & 2 & $125.75 \pm 23.97$ & 2 & 0 & 0 \\
\hline Gurjar & 1 & 139.9 & 1 & 0 & 0 \\
\hline Jamadar & 1 & 137.9 & 1 & 0 & 0 \\
\hline Khatri & 1 & 156.3 & 1 & & 0 \\
\hline
\end{tabular}


Table 4: Mean \pm SD for various variables and geographical distribution.

\begin{tabular}{|llll|}
\hline Variable & Tier $\mathbf{1}$ & Tier $\mathbf{2}$ & Tier $\mathbf{3}$ \\
\hline Age $($ years $)$ & $61.96 \pm 11.18$ & $60.96 \pm 8.73$ & $58.36 \pm 8.5$ \\
\hline Triglycerides $(\mathrm{mg} / \mathrm{dl})$ & $166.91 \pm 59.82$ & $160.83 \pm 90.16$ & $180.94 \pm 86.41$ \\
\hline Total cholesterol $(\mathrm{mg} / \mathrm{dl})$ & $138.67 \pm 39.22$ & $125.44 \pm 31$ & $140.08 \pm 37.35$ \\
\hline HDL $(\mathrm{mg} / \mathrm{dl})$ & $41.10 \pm 9.25$ & $38.27 \pm 9.13$ & $39.04 \pm 9.91$ \\
\hline LDL $(\mathrm{mg} / \mathrm{dl})$ & $64.20 \pm 33.17$ & $56.53 \pm 23.93$ & $65.57 \pm 30.3$ \\
\hline VLDL $(\mathrm{mg} / \mathrm{dl})$ & $33.38 \pm 11.96$ & $32.16 \pm 18.03$ & $36.04 \pm 17.36$ \\
\hline
\end{tabular}

Table 5: Significant differences among ethnic groups according to unpaired t-test and $P$ values.

\begin{tabular}{|ll|ll|}
\hline Parameter & Group-1 & Group-2 & P value \\
\hline Number of drugs & Hindus $(3.86 \pm 1.067)$ & Non-Hindus $(5.13 \pm 1.24)$ & 0.0016 \\
\hline Number of FDCs & Hindus $(1.16 \pm 0.75)$ & Non-Hindus $(1.75 \pm 1.5)$ & 0.0493 \\
\hline \multirow{2}{*}{ Age (years) } & Patel $(57.19 \pm 9.15)$ & Vaishnava $(66.14 \pm 2.34)$ & 0.0162 \\
\cline { 2 - 4 } & Prajapati $(57.75 \pm 2.87)$ & Vaishnava $(66.14 \pm 2.34)$ & 0.0005 \\
\hline \multirow{4}{*}{ Number of FDCs } & Patel $(0.89 \pm 0.64)$ & Rabari $(2 \pm 1)$ & 0.0114 \\
\cline { 2 - 4 } & Patel $(0.89 \pm 0.64)$ & Rajput $(1.38 \pm 0.74)$ & 0.0179 \\
\cline { 2 - 4 } & Rajput $(1.38 \pm 0.74)$ & Vaishnava $(0.71 \pm 0.49)$ & 0.035 \\
\cline { 2 - 4 } & Rabari $(2 \pm 1)$ & Vaishnava $(0.71 \pm 0.49)$ & 0.0213 \\
\hline Triglycerides $(\mathrm{mg} / \mathrm{dl})$ & Patel $(177.63 \pm 109.2)$ & Rajput $(165.07 \pm 66)$ & 0.0489 \\
\hline
\end{tabular}

\section{DISCUSSION}

The concept of ethnicity is a vital tool to provide important information about shared exposures for individuals with a similar geographical origin, culture, traditions, language and access to health services. ${ }^{7}$ There has been a substantial debate on the concepts of ethnicity and race within health services. ${ }^{8}$ Significant variations in dyslipidemia prevalence, its relation to $\mathrm{CAD}$ and response to hypolipidaemic drugs have been noticed across ethnic groups. ${ }^{9}$ So, a tailored disease management becomes necessary. While South Asians account for only $25 \%$ of the world's population, they account for a disproportionate $60 \%$ of the world's cardiovascular disease burden and this ancestry is now considered a strong independent risk factor for CAD. Small dense LDL and high serum Lipoprotein(a) may contribute to a greater risk among them.

When compared to Western populations, Indians tend to have higher triglyceride levels and lower HDL levels but the total cholesterol levels are generally lower than in the US or the UK populations. Low HDL levels and hypertriglyceridemia are metabolically interrelated and this combination is known as atherogenic dyslipidemia. ${ }^{10}$ In recent times, there has been an increase in the incidence of atherogenic dyslipidaemia in Indians compared to Western population, which may be due to adverse lifestyle changes such as physical inactivity, diet poor in polyunsaturated fatty acids (PUFA) and a higher genetic predisposition. ${ }^{11}$

In view of the recommendations from different international organizations and especially the absence of
India-specific guidelines, the approach followed by Indian doctors to manage dyslipidaemia patients in routine clinical practice remains uncertain and currently, there is lack of data on the same.

\section{Demographic details}

The mean \pm SD age of the enrolled patients was $60.5 \pm 9.85$ years, comparable to a study conducted in Pune where the mean age \pm SD was found to be $60.9 \pm 12.4$ years. ${ }^{12}$ The male:female ratio of the patients included in this study was 4.9:1 indicating a clear preponderance of CAD in males. This value is much higher than 2.6, the gender ratio of the patients in another study. ${ }^{12}$

\section{Evaluation of drug prescription pattern}

The mean number of drugs per prescription \pm SD was 3.94 \pm 1.12 which is higher than the value obtained in the study carried out in Southern India, lower than that obtained in the study undertaken in Jammu and Kashmir and much lower than that obtained in another study conducted at Ahmedabad. ${ }^{13-15}$ The hypolipidaemic drugs prescribed included drugs from the statins or fibrates group. Statins were combined with drugs like Aspirin, Clopidogrel or a Fibrate. The most commonly prescribed hypolipidaemic drug was Rosuvastatin, in contrast to Atorvastatin being the most commonly prescribed hypolipidaemic drug in the studies conducted in Southern India, Jammu and Kashmir and Ahmedabad. ${ }^{13-15}$

Ezetimibe, a cholesterol absorption inhibitor was also prescribed in a fraction of patients in another study conducted 
at Ahmedabad ${ }^{15}$ in contrast to not a single patient prescribed with this drug in this study. Rosuvastatin and Atorvastatin are fluorine containing congeners having plasma half-lives of 14 and 19 hours respectively. Rosuvastatin causes a rise in HDL by $15-20 \%$ which is greater than other statins; also, it is more potent than Atorvastatin on a dose to dose basis. In our study the prescription of Rosuvastatin among all other statins has dominated. Rosuvastatin is a newer statin but its efficacy is not superior. Atorvastatin on the other hand is documented to have additional antioxidant property which may prove to be useful in CAD patients. Here the authors will not like to hesitate in stating that new drugs are considered as superior and prescribed more often. This practice needs to be modified.

It was also noted that among hypolipidaemic drug classes all the prescriptions involved statins. No non-statin drug therapies except Fenofibrate were prescribed which questions the efficacy and tolerability of other hypolipidaemic agents like bile acid sequestrants, Niacin and Ezetimibe.

\section{Lipid profile patterns and ATP III risk assessment}

All lipid levels except triglycerides and HDL were controlled with drug therapy in most patients. Low HDL is an independent risk factor for CAD and classically statins cannot increase HDL beyond 5-15\%. Despite low HDL levels in a majority of patients, no HDL raising drug like Nicotinic acid was precribed to any patient.

Atherogenic dyslipidemia (AD) was present in $35.77 \%$ patients which is lower than its prevalence $(41.3 \%)$ observed in a study conducted at Pune and is higher than that in another study (13\%). ${ }^{16}$ Majority of these patients belonged to the age group of 51-60 years (43.2\%) which is in contrast to the study conducted at Pune which concluded that $\mathrm{AD}$ had no association with age.

\section{Ethnicity and its association with lipid values and hypolipidaemic drug utilization patterns}

Lipid profile patterns were poorer in Hindus compared to patients from other religions. In majority of the castes, triglyceride levels were not controlled even after treatment. On the whole Jains and Patels had an overall poorer lipid profile picture compared to other castes. Most of the lipid values were higher in patients of Tier-3 cities which may be due to diet containing more saturated and trans fats, obesity, lack of exercise, smoking and co-morbidities like diabetes mellitus. Majority of the elderly patients belonged to Tier-1 city which suggests the better healthcare facilities and a higher life expectancy in large cities. Atorvastatin was prescribed more to the patients from Tier-3 cities which may be due to less cost.

Study was done for the first time to associate religion, caste and geographical distribution to lipid profile values. ATPIII guidelines by NCEP were used to classify the lipid levels and to assess the risk. Drug utilization pattern in CAD was well studied including the major hypolipidaemic drugs used and their effects on various lipid parameters.
This was a limited duration pilot study and the low sample size is due to short term study permitted by ICMR. The sample size consisted predominantly of Hindu patients. Hence the comparison between Hindus and other religious sectors could not be reliable and robust although the investigator did try to compare a few aspects. Similarly, there was not enough representation of the castes, predominant ones were Brahmins, Patels and Rajputs. South Asian patients from 4 states of India were included. However, this will serve as a benchmark for larger studies.

\section{CONCLUSION}

Ethnic differences do exist in lipid levels among CAD patients. Hindu patients in this study showed a poorer lipid profile while among the castes, Jains and Patels fared poorly. Patients from Tier-3 cities also showed greater dyslipidemia manifestations. High triglyceride levels and lower HDL values were quite common, showing that atherogenic dyslipidemia is on a rise in the Indian population. The differences in prevalence rates of CAD in various ethnic groups cannot be explained completely on the basis of classical risk factors and support its multifactorial causation.

They could be ascribed to sedentary lifestyle, obesity, heredity, dietary factors, lack of exercise, smoking and comorbidities. Non-statin therapies were hardly used which questions their status in dyslipidemia. Extensive prescription of newer drugs and zero prescription of drugs by generic name were also observed.

\section{ACKNOWLEDGEMENTS}

This project was supported by the Indian Council of Medical Research (ICMR). The authors thank the Dean of their college and the Superintendent who allowed to conduct the research in the hospital premises, the Department of Cardiology for the endless support and guidance at every step of the project and the Department of Pharmacology who provided insight and expertise that greatly assisted the research.

\section{Funding: Indian Council of Medical Research (ICMR) Conflict of interest: None declared \\ Ethical approval: The study was approved by the Institutional Ethics Committee}

\section{REFERENCES}

1. Mendis S, Puska P, Norrving B. Global atlas on cardiovascular disease prevention and control/edited by: Shanthi, Mendis et.al. Geneva: World Health Organization. 2011. Available at http://www.who.int/iris/handle/10665/44701. accessed 06 June 2019.

2. Carleton RA, Dwyer J, Finberg L, Flora J, Goodman DS, Grundy SM, et al. Report of the Expert Panel on Population Strategies for Blood Cholesterol Reduction. A statement from the National Cholesterol 
Education Program, National Heart, Lung, and Blood Institute, National Institutes of Health. Circulation. 1991 Jun;83(6):2154-232.

3. Friedewald WT, Levy RI, Fredrickson DS. Estimation of the concentration of low-density lipoprotein cholesterol in plasma, without use of the preparative ultracentrifuge. Clin Chem. 1972 Jun;18(6):499-502.

4. Patel J, Sheehan V, Gurk-Turner C: a new type of lipid lowering agent. Proc. 2003;16(3):354-8.

5. Iyengar S, Puri R, Narasingan S. Lipid Association of India Expert Consensus Statement on Management of Dyslipidemia in Indians 2016: Part 1-Executive summary. J Clin Prev Cardiol. 2016;2(2);134.

6. Third Report of the National Cholesterol Education Program (NCEP) Expert Panel on Detection, Evaluation, and Treatment of High Blood Cholesterol in Adults (Adult Treatment Panel III) final report. Circulation. 2002 Dec17;106(25):3143-421.

7. Mathur, R; (2015) Ethnic inequalities in health and use of healthcare in the UK: how computerized health records can contribute substantively to the knowledge base. PhD thesis, London School of Hygiene \& Tropical Medicine. Available at: http://researchonline.lshtm.ac.uk/2478832/.

8. Lip GY, Barnett AH, Bradbury A, Cappuccio FP, Gill PS, Hughes E, et.al. Ethnicity and cardiovascular disease prevention in the United Kingdom: a practical approach to management. J Hum Hypertens. 2007 Mar;21(3):183-211.

9. Frank AT, Zhao B, Jose PO, Azar KM, Fortmann SP, Palaniappan LP. Racial/ethnic differences in dyslipidemia patterns. Circulation. 2014 Feb 4;129(5):570-9.

10. Grundy SM. Atherogenic dyslipidemia: Lipoprotein abnormalities and implications for therapy. Am J Cardiol. 1995;75:45B-52B.
11. Manjunath CN, Rawal JR, Irani PM, Madhu K. Atherogenic dyslipidaemia. Indian $\mathrm{J}$ Endocrinol Metab. 2013 Nov-Dec;17(6):969-76.

12. Mahalle N, Garg MK, Naik SS, Kulkarni MV. Study of pattern of dyslipidemia and its correlation with cardiovascular risk factors in patients with proven CAD. Indian J Endocrinol Metab. 2014 Jan;18(1):48-55.

13. Sangeetha Raja. Prescription pattern of hypolipidemic drugs, PCOS patients. J Clin Diagn Res. 2014 Apr;8(4):HC01-3.

14. Gupta S, Kumar R, Kumar D, Bhat S, Kumar D, Bhat NK et al. Study of prescribing patterns of hypolipidemic agents in a tertiary care teaching hospital in North India. Natl J Physiol Pharm Pharmacol. 2017;7(2):198-202.

15. Patel KP, Joshi HM, Khandhedia C, Shah H, Shah KN, Patel VJ. Study of drug utilization, morbidity pattern and cost of hypolipidemic agents in a tertiary care hospital. Int J Basic Clin Pharmacol. 2013;2:470-5.

16. C.Lahoz, J.M.Mostaza, S.Tranche. Atherogenic dyslipidemia in patients with established coronary artery disease. Nutr Metab Cardiovasc Dis. 2012 Feb;22(2):103-8.

Cite this article as: Bharadwaj SP, Patel AT, Rana DA, Malhotra SD, Patel TM. Linking of different ethnicities, races and religions to lipid profile patterns and hypolipidaemic drug usage patterns in coronary artery disease patients. Int J Basic Clin Pharmacol 2019;8:1707-12. 